\title{
Mandela: histoire extraordinaire d'une guerre civile évitée
}

\section{Jean Martin}

1 Mandela N. Pensées pour moi-même - Le livre autorisé de citations. Publié sous la direction de Sello Hatang et Sahm Venter. Editions de la Martinière, 2011.

\section{jean.martin[at]saez.ch}

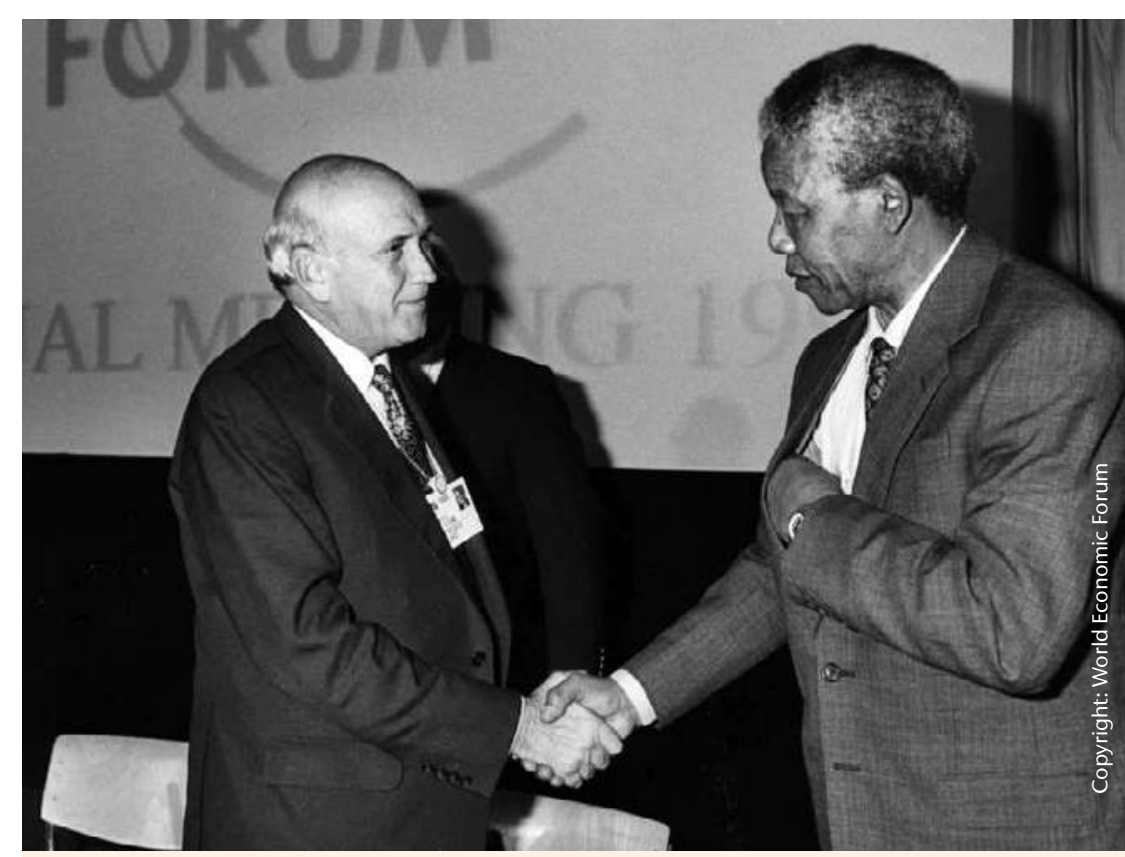

Le président Nelson Mandela et son prédécesseur Frederik De Klerk se serrent la main à la réunion annuelle du World Economic Forum à Davos, en 1992.

\begin{abstract}
S'intéressant à l'Afrique du Sud de la fin du XXe siècle, on voit que les ressorts étaient montés pour une guerre civile qui aurait été terrible. Dans un contexte d'oppression majeure et de révolte, et grâce aussi à la lucidité de responsables blancs, Nelson Mandela a réussi à faire en sorte que cette guerre n'ait pas lieu. Les guerres évitées sont vite oubliées... leur histoire pourtant devrait vivement retenir l'attention. On aurait de l'emploi aujourd'hui pour des géants de ce calibre alors que dans nombre d'endroits se perpétuent les antagonismes, crises, guerres - civiles ou pas, des abcès qui laissent désarmé. Quelques miracles du type Mandela seraient les bienvenus.

La bibliographie et les biographies le concernant sont riches. Je viens de lire un recueil de près de 500 pages publié par le Centre pour la mémoire de la Fondation Nelson Mandela [1]. La plupart des citations qui suivent sont des années 1990 (après 27 ans de prison, il en est sorti en février 1990) jusqu'à 2008 (né en 1918, il est mort en décembre 2013). Quelques-unes sont antérieures, notamment de l'époque des procès contre lui en 1962 et 1964. Je les ordonne en plusieurs registres.
\end{abstract}

\section{Lutte contre l'oppression}

«Même si nos espérances ne se réalisent pas, nous devons garder à l'esprit que dans la vie le plus glorieux n'est pas de ne jamais tomber, mais de toujours se relever.»

«J'ai appris que le courage n'était pas de ne pas avoir peur, mais d'en triompher. L'homme courageux est celui qui apprivoise la peur»

«ll y a une contradiction inhérente aux organisations politiques: le leader doit maintenir vives les forces qui ont permis à l'organisation d'exister mais il ne peut le faire qu'en autorisant les dissensions.» Mais il dit ailleurs: «Les divergences théoriques entre combattants de l'oppression sont un luxe que nous ne pouvons pas nous permettre...»

«Nous ne voulons pas la liberté sans le pain, de même que nous ne voulons pas le pain sans la liberté.»

«L'oppression déshumanise l'oppresseur autant qu'elle fait souffrir l'opprimé. [...] Je savais d'ores et déjà que l'oppresseur doit être libéré, comme l'opprimé. Un homme qui enlève à un autre sa liberté est prisonnier de la haine, il est enfermé derrière les barreaux des préjugés.»

\section{Politique}

«L'arme la plus puissante n'est pas la violence, mais la discussion.»

«J'ai appris qu'humilier quelqu'un c'est lui faire subir un sort cruel. Déjà enfant, je battais mes adversaires sans les déshonorer.»

«Une partie de la grandeur de ce pays réside dans la manière dont nous identifions, admettons et discutons les problèmes et les défis qui se présentent à nous [...] Nous avons choisi la route de la négociation et du compromis. Les Sud-Africains sont passés par-dessus des divisions vieilles de plusieurs siècles et ont évité le bain de sang que la plupart des observateurs jugeaient inévitable.»

«Pour réaliser la transition, nous n'avons rien détruit ou brisé sans raison. Nous avons dans bien des cas repris les anciennes formes en y ajoutant les nouvelles valeurs d'humanité et de solidarité.»

«Les êtres humains ont des côtés forts et des points faibles. Notre devoir est d'œuvrer avec eux tels qu'ils sont, et non parce qu'on les prend pour des anges. L'important est de se souvenir qu'aucune personne ne peut tout faire toute seule.»

«Il est crucial de s'entourer, à la fois dans les structures et dans le travail individuel, de gens qui sont forts et qui résisteront si vous vous trompez.» Ce qui me rappelle (J.M.) la phrase d'un Conseiller d'Etat sous lequel j'ai servi, disant qu'on ne peut s'appuyer que sur ce qui résiste.

«S'il y a une chose que je répète, c'est que les gens ne devraient pas entretenir des espérances exagérées, 
qu'on ne peut pas régler leurs problèmes en une nuit.»

Rappel d'un fait qui est à l'origine de tant des tribulations actuelles de l'Afrique: «Les frontières nationales créées artificiellement à l'époque coloniale ont séparé des tribus et des clans, coupé les routes migratoires des animaux, fragmenté les écosystèmes et conduit à la destruction de la biodiversité».

\section{Négociation et raison - Nécessité du compromis}

«Dans pratiquement toutes les parties du monde, des êtres humaines trouvent des raisons de recourir à la violence et à la force pour régler des différends que nous aurions dû tenter de résoudre par la négociation, le dialogue et la raison.»

«Il faut chercher des compromis pour tous les problèmes, tant que ces compromis sont dans l'intérêt non d'une partie de la population mais de tout le pays.»

«La plus importante leçon apprise au cours de ma vie consacrée au combat pour la liberté, c'est que dans tout conflit arrive un moment où aucun des deux camps ne peut plus prétendre avoir tout à fait raison. La recherche de compromis est alors la seule solution.»

«Quand vous négociez, vous devez penser à la réconciliation, pas à lancer des ultimatums. [...] Quand on ne veut pas d'un compromis, il ne sert à rien de négocier.»

«Rien de plus néfaste que de faire une demande dont on sait qu'elle n'a aucune chance d'aboutir.»

«Parmi les principes qu'a suivis le mouvement de libération, il y avait celui que nous devions en sortir plus forts et plus unis, qu'il ne devait y avoir ni gagnants ni perdants.»

\section{Ecoute, attention à l'autre - Vivre ensemble}

«Nos différences sont notre force en tant qu'espèce réunie dans une communauté mondiale. On s'enrichit beaucoup en rencontrant des personnes qui ont eu des chemins de vie différents.»

«Le défi le plus fondamental dans cette ère post-apartheid est de faire de l'attention à autrui la caractéristique principale de notre société [...]. Un simple sourire, un salut ou une main tendue disent: Je ne suis rien sans vous, je suis plus riche grâce à vous, je vous respecte tel que vous êtes.»»

«'en suis venu à accepter que je n'avais pas le droit de juger les autres en fonction de mes propres coutumes; que mépriser les autres parce qu'ils n'observaient pas telle ou telle coutume était une forme dangereuse de chauvinisme.»

"La démocratie et les droits de l'homme sont inséparables.»

«La liberté de parler de la liberté constitue l'essentiel même de la légitimité de l'ordre constitutionnel [...] - qui doit en outre reconnaître le droit à la contestation. Jouir de la liberté oblige à créer les conditions pour que les autres en jouissent.»

«Dans la position qui est la mienne (Président du pays), la tâche principale est de rassembler les diverses factions, donc d'écouter très attentivement quand quelqu'un vient m'exposer un problème» (plus on est dans une fonction élevée, plus il faut écouter - conviction de J.M.).

«Si vingt-sept ans de prison nous ont servi à quelque chose, c'est à profiter du silence de la solitude pour comprendre que les mots sont précieux.»

\section{Surmonter la haine et la crainte de l'autre}

«Quand nous nous sommes rencontrés [avec les responsables politiques blancs, vers 1990], nous avons découvert qu'en fait c'étaient des gens normaux, qui répondaient de manière constructive, avec humanité. Heureusement, parmi nos ennemis, certains ont eu le cran de dire «Oublions le passé.` Alors nous nous sommes assis et nous avons parlé. Cela a été une grande source de fierté.»

«Nous avons donné tort à tous les prédicteurs d'apocalypse, simplement parce que nous étions disposés à accepter la capacité inhérente de l'autre à la bonté.»

«Nous pensons toujours que l'autre est le diable avant de l'avoir rencontré» - à propos d'une rencontre avec Madame Verwoerd, épouse d'un président de l'époque de l'apartheid.

«On fait de grands progrès quand on part du principe simple qu'on à face à soi un homme intègre.»

«Il m'avait fait perdre le contrôle de mes nerfs et je l'ai vécu comme une défaite.»

Education: «Les gens apprennent à haïr, et si on leur apprend à haïr, on peut aussi leur apprendre à aimer.»

«Chez les gens qui se sont retrouvés en prison alors qu'ils essayaient de transformer la société, le pardon est naturel. Ils n'ont pas de temps à perdre à se venger.»

\section{Soins de santé et besoins de base}

«La liberté ne se comprend pas en termes de positions de pouvoir. Il faut la comprendre comme la transformation de la vie pour tous les gens de peu.»

«La pauvreté massive et les inégalités obscènes sont de tels fléaux, quand pourtant le monde peut se vanter de stupéfiants progrès, qu'il faut les classer dans les maladies sociales, aux côtés de l'esclavage et de l'apartheid.»

«Il y a quatre choses élémentaires que veut la masse des gens qui composent une société: vivre dans un environnement sûr; pouvoir travailler et gagner son pain; avoir accès à des soins de qualité; offrir une éducation correcte à ses enfants.»

«Il ne peut y avoir de paix tant que notre peuple manque de structures sociales de base, comme par exemple l'accès la médecine.»

Nelson Mandela s'est beaucoup engagé pour la prévention du sida et les soins aux personnes touchées (alors que son successeur Thabo Mbeki est de ceux qui ont nié publiquement et de manière répétée la gravité du problème): «Nous devons offrir aux gens un envi- 
ronnement qui les aide à se protéger grâce à un accès facilité aux préservatifs, aux médicaments, aux tests et à des conseils. Il faut s'élever contre la stigmatisation, la condamnation et le déni qui ont jusqu'ici été associés à cette épidémie.»

«Rien ne requiert plus notre attention que les orphelins du sida, si souvent rejetés et ostracisés; ces enfants qui souffrent physiquement, socialement et moralement des conséquences de comportements sur lesquels ils n'ont eu aucun contrôle.»

«Mon fils est mort du sida» (conférence de presse, janvier 1995).

«Pour chaque femme agressée nous rabaissons notre humanité. Pour chaque femme forcée par un homme à une relation sexuelle, nous rabaissons notre dignité. Chaque fois que nous gardons le silence, nous conspirons contre les femmes.»

«Entendons la voix des femmes africaines dans la lutte contre le sida. Que leurs voix se fassent entendre jusqu'aux centres du pouvoir.»

«Nous soutenons la notion que, si les enfants ont besoin d'être guidés, ils ont aussi le droit inaliénable de choisir ce qu'ils veulent devenir, et que cela n'est possible que si on leur donne l'espace pour vivre leurs rêves.»

\section{Les anciens, les origines}

«Je me sens tenu de rendre hommage à mes coutumes et à mes traditions, pourvu qu'elles nous permettent de rester ensemble et n'entrent pas en conflit avec les objectifs de la lutte contre l'oppression.»

«La science a permis d'acquérir beaucoup de connaissances mais même les générations d'aujourd'hui savent que l'expérience des anciens est inestimable. Une société qui n'accorde aucune valeur à ses anciens nie ses racines et compromet son avenir.» Ceci alors que:«Pendant trois siècles, les Blancs ont essayé de dire aux Noirs qu'ils n'avaient pas d'histoire, pas de civilisation et d'identité dont ils pouvaient s'enorgueillir.»

«Le passé est une riche ressource dans laquelle nous pouvons puiser pour prendre des décisions concernant l'avenir, mais il ne dicte pas nos choix. Nous devons prendre ce qu'il y a de bon et laisser le mauvais de côté.»

«L'esprit d'ubuntu, ce sentiment profondément africain d'appartenance à l'humanité des autres, la reconnaissance que nous sommes des individus seulement grâce aux autres individus, s'est complètement agrégé à notre quête commune d'un monde meilleur.»

\section{Porter un regard sur ce qui a été fait et pourquoi}

«C'est un honneur pour l'homme de faire son devoir sur terre, quelles que soient les conséquences.»

«C'est la différence faite dans la vie des autres qui définit le sens de la vie que nous avons menée.»

«Un des plus grands héritages de la lutte menée par Steve Biko - et la raison pour laquelle il est mort [en prison suite à des violences] - est la fierté qu'il a fait naître chez les victimes de l'apartheid.»

«Je me suis consacré à la défense du peuple africain. J'ai combattu la domination blanche et j'ai combattu la domination noire. J'ai chéri l'idéal d'une société démocratique libre, non raciale et non sexiste, dans laquelle toutes les personnes vivraient avec des chances égales.»

«Ce ne sont pas forcément les hommes et les femmes en situation de responsabilité, mais les hommes et les femmes ordinaires, qui comprennent que les plus grands défis sont pauvreté, illettrisme, maladie... Ce sont eux mes héros.»

«Je me risque à dire qu'il y a une part de bonté naturelle en l'homme, qu'on peut attribuer entre autres à la conscience sociale que nous possédons tous.»

"Cela m'a beaucoup inspiré de servir une nation qui a contribué à redonner au monde l'espoir que tous les conflits, aussi insolubles qu'ils paraissent, pourront connaitre une issue pacifique. Nous symbolisons le fait qu'il est possible de faire le bien entre êtres humains prêts à se faire confiance.»

«Dans le monde moderne, aucun pays ne peut conduire tout seul ses affaires. Les problèmes auxquels nous faisons face demandent de tels moyens qu'aucune nation ne peut les résoudre sans les autres.»

«Voyant des fermiers afrikaners discuter dans une queue avec leurs employés, dans une ambiance détendue, on comprend qu'une nouvelle Afrique du Sud est née.»

Et un regard sur soi, avec un peu d'auto-dérision «Le plus difficile n'est pas de changer la société, mais de se changer, soi.»

«Les gens ont l'impression que je vois toujours le bien dans les gens. C'est une critique que je prends en compte et j'essaie de me corriger, mais je pense que c'est une faiblesse profitable.»

«Médire en parlant des autres est certainement un vice; quand on parle de soi, c'est une vertu.»

«Ma foi, ça fait du bien aux gens de parler de moi comme d'un héros. Ce n'est pas vraiment de moi qu'ils parlent. Je suis comme une patère sur laquelle on accroche toute l'adulation.»

«Je suis de ceux qui possèdent des bribes d'information sur une grande variété de sujets, mais à qui il manque une connaissance profonde et pointue d'un domaine donné.»

"Quand je resterai assis à Qunu [son lieu d'origine] et que je deviendrai aussi vieux que ses collines, je continuerai à entretenir l'espoir qu'apparaissent toujours dans le monde des dirigeants qui ne laissent personne être bafoué comme nous l'avons été, être réduit à la faim comme nous l'avons été, être dépouillé de sa dignité comme nous l'avons été.»

«En dehors de l'affection de ma femme et de ma famille, peu de choses m'ont plus inspiré que de savoir que les gens ne nous oublient pas.» 